\title{
Clinical outcome of subclinical Cushing's syndrome after surgical and conservative treatment
}

\author{
Itaru Akaza, Takanobu Yoshimoto, Fumiko Iwashima, Chisato Nakayama, Masaru Doi, Hajime Izumiyama \\ and Yukio Hirata
}

\begin{abstract}
Subclinical Cushing's syndrome (SCS), a subtle cortisol hypersecretion from an adrenal tumor, may be a common adrenal disease. However, the cardiovascular prognosis and the optimal surgical and conservative treatment in SCS remain elusive. The present study was undertaken to evaluate the prevalence of cardiovascular risk factors in 16 SCS cases, their relationships to cortisol secretory activities, and the clinical outcome after surgical and medical treatment. The prevalence of hypertension, impaired glucose tolerance (IGT), diabetes mellitus (DM), dyslipidemia and obesity in our SCS cases were $56 \%, 50 \%, 50 \%$, and $19 \%$, respectively, and $75 \%$ of cases were associated with two or more cardiovascular risk factors. In our series, 24-h urinary free-cortisol excretion showed a significant positive correlation with $\mathrm{HbA1C}$ and a negative correlation with high-density lipoprotein-cholesterol, but no correlation with age, body mass index, blood pressure or glycemic and lipid profile was found. Eight cases underwent unilateral adrenalectomy (operated (OP) group); the remaining eight cases were a conservative-treatment group (non-OP group). The number of cardiovascular risk factors decreased significantly in the OP group, but not in the non-OP group. In terms of differential changes in risk factors between the groups, more significant improvements of hypertension, dyslipidemia and IGT/DM were observed in the OP group than in the non-OP group. In conclusion, the present study showed the increased prevalence of cardiovascular risk factors in SCS patients with mild hypercortisolism related to impaired glucose/lipid metabolism. Adrenalectomy decreased accumulated cardiovascular risk factors in certain SCS patients, suggesting the possible involvement of mild hypercortisolism in the development of cardiovascular risk factors in SCS.
\end{abstract}

Hypertension Research (2011) 34, 1111-1115; doi:10.1038/hr.2011.90; published online 21 July 2011

Keywords: adrenalectomy; cardiovascular risk factors; subclinical Cushing's syndrome

\section{INTRODUCTION}

The term subclinical Cushing's syndrome (SCS) is commonly used to define a condition characterized by subtle cortisol hypersecretion from an adrenal tumor without signs or symptoms of overt Cushing's syndrome (CS). ${ }^{1}$ SCS accounts for $\sim 5-20 \%$ of incidentally discovered adrenal masses (adrenal incidentalomas). ${ }^{2,3}$ Given that $4 \%$ of subjects older than 60 years of age harbor such adrenal tumors, SCS is considered a potentially common adrenal disease. ${ }^{4,5}$

It has been shown that SCS is associated with insulin resistance, hypertension, obesity, dyslipidemia, impaired glucose tolerance (IGT) and diabetes mellitus (DM) as frequently as is overt CS. ${ }^{6-8}$ Although it has been well recognized that overt CS patients have a higher rate of mortality due to cardiovascular complications, such as myocardial infarction, congestive heart failure and stroke, ${ }^{9,10}$ the cardiovascular prognosis of SCS remains undetermined. Several previous studies have shown the improvement of hypertension after surgical treatment in SCS; ${ }^{8,11-13}$ however, the outcome of other cardiovascular risk factors, such as diabetes, dyslipidemia and obesity, remains obscure. Because there have been no large studies on the cardiovascular prognosis in
SCS, the clinical outcome achieved by surgical and medical management of SCS after long-term follow-up is open to debate. ${ }^{14}$

To address the issue, the present retrospective study was undertaken to evaluate the prevalence of cardiovascular risk factors in 16 patients with SCS, their relationships to cortisol secretory activity, and the associated clinical outcomes after surgical and medical treatment.

\section{METHODS}

Study population

In all, 16 of 168 patients with adrenal tumors (9.5\%) who were diagnosed as SCS at Tokyo Medical and Dental University Hospital during 2002-2008 were retrospectively studied. This study was approved by the Ethics Committee of our institute. Informed consent was obtained from each patient.

The diagnosis of SCS was made on the basis of the diagnostic criteria proposed by the Research Committee for Adrenal Diseases provided by the Japanese Ministry of Health, Labor and Welfare. ${ }^{15}$ These criteria include the presence of adrenal incidentaloma, the lack of Cushingoid features and normal basal serum cortisol levels with no suppression by an overnight low-dose ( $>3 \mu \mathrm{g} \mathrm{dl} l^{-1}$ with $\left.1 \mathrm{mg}\right)$ and a high-dose $\left(>1 \mu \mathrm{g} \mathrm{dl}{ }^{-1}\right.$ with $\left.8 \mathrm{mg}\right)$ dexamethasone suppression test, and at least one of the following additional endocrine 
abnormalities: (1) suppressed plasma adrenocorticotropic hormone (ACTH) $\left(<10 \mathrm{pg} \mathrm{ml}^{-1}\right)$ and/or decreased response to corticotropin-releasing hormone (CRH) stimulation ( $<150 \%$ increase compared with baseline), (2) loss of cortisol diurnal rhythm ( $>5 \mu \mathrm{g} \mathrm{dl}^{-1}$ at midnight), decreased serum dehydroepiandrosterone sulfate levels (less than age- and sex-matched reference values), and (4) unilateral radioactive uptake, as determined by adrenal scintigraphy.

Surgical treatment is recommended for the patients with multiple (two or more) cardiovascular risk factors and/or those who desire and agree to undergo surgery, whereas medical treatment is recommended for the patients who are unwilling to undergo surgery regardless of the presence or the absence of cardiovascular risk factors.

\section{Cardiovascular risk factors}

Hypertension is defined as diastolic blood pressure $>90 \mathrm{~mm} \mathrm{Hg}$ and/or systolic blood pressure $>140 \mathrm{~mm} \mathrm{Hg}^{16}$ obesity as body mass index $>25 \mathrm{~kg} \mathrm{~m}^{-2}$; IGT as fasting plasma glucose (FPG) $110-125 \mathrm{mg} \mathrm{dl}^{-1}$ and/or $2 \mathrm{~h}$ plasma glucose $140-199 \mathrm{mg} \mathrm{dl}^{-1}$ on the $75 \mathrm{~g}$ oral glucose tolerance test (OGTT); type $2 \mathrm{DM}$ as FPG $\geqslant 126 \mathrm{mg} \mathrm{dl}^{-1}$ and/or $2 \mathrm{~h}$ plasma glucose $\geqslant 200 \mathrm{mg} \mathrm{dl}^{-1}$ on the $75 \mathrm{~g}$ OGTT or elevated HbAlc $(\geqslant 6.1) ; 17$ and dyslipidemia as triglyceride (TG) $\geqslant 150 \mathrm{mg} \mathrm{dl}^{-1}$, high-density lipoprotein-cholesterol (HDL-C) $<40 \mathrm{mg} \mathrm{dl}^{-1}$, or low-density lipoprotein-cholesterol (LDL-C) $\geqslant 140 \mathrm{mg} \mathrm{dl}^{-1}$. 18 Patients on medication for hypertension, type $2 \mathrm{DM}$ and dyslipidemia were included as having the respective risk factors.

\section{Follow-up study}

Eight patients underwent surgical adrenalectomy (the operated (OP) group consisted of six patients with multiple cardiovascular risk factors and two patients who desired surgical treatment), whereas the remaining eight patients were treated with conservative management (non-OP group). Blood pressure (systolic, diastolic), glycemic profile (FPG, immunoreactive insulin (IRI), homeostasis model assessment insulin resistance index, HbAlc, 75 g OGTT), lipid profile (TG, HDLC, LDL-C) and obesity (body mass index) were evaluated before and after surgery in the OP group. The follow-up period was $28 \pm 7$ months and $40 \pm 19$ months for the OP and non-OP groups, respectively.

\section{Clinical outcome}

Changes in blood pressure, body weight, and the glycemic and lipid profiles during the follow-up period were evaluated as improved, unchanged or deteriorated. Improvement of hypertension is defined as normalized blood pressure (systolic blood pressure $<140 \mathrm{~mm} \mathrm{Hg}$ and diastolic blood pressure $<90 \mathrm{~mm} \mathrm{Hg}$ ) and discontinuation or reduction of the use of anti-hypertensive drugs (number or dose). Deterioration was defined as aggravated hypertension (systolic blood pressure $>140 \mathrm{mmHg}$ and/or diastolic blood pressure $>90 \mathrm{~mm} \mathrm{Hg}$ ) and/or increased dose or number of anti-hypertensive drugs, respectively. Improvement of IGT/DM was defined as normoglycemia/IGT on OGTT, $>0.3 \%$ decrease in HbAlc levels or discontinuation/decrease in the number or dose of insulin/oral hypoglycemic agents. Deterioration was defined as the development of IGT/DM on OGTT, a $>0.3 \%$ increase in HbAlc levels, or initiation or increase in the dose of insulin/oral hypoglycemic agents treatment. ${ }^{12}$ Improvement of dyslipidemia was defined as normalized plasma lipid levels ( $\mathrm{TG}<150 \mathrm{mg} \mathrm{dl}^{-1}$, HDL-C $\geqslant 40 \mathrm{mgdl}^{-1}$ and LDL-C $<140 \mathrm{mg} \mathrm{dl}^{-1}$ ) or discontinuation or decrease in the number or dose of anti-dyslipidemia drugs). Deterioration was defined as aggravated dyslipidemia $\left(\mathrm{TG} \geqslant 150 \mathrm{mg} \mathrm{dl}^{-1}\right.$, HDL-C $<40 \mathrm{mg} \mathrm{dl}^{-1}$ and/or LDL-C $\geqslant 140 \mathrm{mg} \mathrm{dl}^{-1}$ ) or initiation or increase in the dose of anti-dyslipidemia drug treatment. Improvement and deterioration of obesity were defined as a $>3 \mathrm{~kg}$ decrease or increase, respectively, in body weight compared with baseline. ${ }^{12} \mathrm{~A}$ lack of 'improvement' or 'deterioration' of cardiovascular risk factors was defined as 'unchanged.'

\section{Hormonal measurements}

Blood samples were withdrawn on the morning after overnight fasting. Plasma ACTH and serum cortisol were measured by immunoradiometric assay (ACTH IRMA Mitsubishi, Mitsubishi Chemical, Tokyo, Japan) and competitive enzyme immunoassay (ST AIA-PACK CORT, Tosoh, Tokyo, Japan), respectively. The serum dehydroepiandrosterone sulfate level was measured by conventional radioimmunoassay.

\section{Statistical analysis}

Data are expressed as the means \pm s.d. or median (interquartile range of 25-75\%), depending on the distribution of data. A log transformation was performed for variables that did not follow a normal distribution. The $\chi^{2}$ test was used to compare proportions of subjects in the two groups. Between-group comparisons were performed using the non-paired $t$-test. The relationship between two continuous variables was evaluated using Pearson's correlation coefficient. Pre- and post-treatment differences were analyzed using Wilcoxon's signed-rank test. $P$-values $<0.05$ were considered significant. All statistical analyses were performed using Windows software Prism 5.0 (GraphPad Software, La Jolla, CA, USA).

\section{RESULTS}

Clinical characteristics of 16 patients with SCS are shown in Table 1. Eight patients underwent unilateral adrenalectomy (OP group), and the remaining eight patients received conservative treatment (non-OP group). Gender, age and the prevalence of cardiovascular risk factors, including hypertension (56\%), DM/IGT (25\%), dyslipidemia (50\%) and obesity (19\%), were evaluated. Fasting insulin, homeostasis model assessment insulin resistance index, serum cortisol levels, plasma ACTH levels and 24-h urinary free-cortisol (UFC) excretion were comparable between both groups, although HbAlc and serum dehydroepiandrosterone sulfate levels were significantly $(P<0.05)$ higher and lower in the OP group than in the non-OP group, respectively. Tumor sizes $(26.3 \pm 8.7 \mathrm{~mm})$ were similar between the two groups.

To determine whether hypercortisolism is related to cardiovascular risk factors, univariate analysis of the relationship between cortisol secretion and various clinical factors was performed. Twenty-fourhour UFC excretion at diagnosis showed a significant $(P<0.05)$ positive correlation with $\mathrm{HbA1c}(r=0.63)$, a negative correlation with HDL-C $(r=-0.56)$ and a negative, although insignificant, correlation with FPG $(r=0.49, P=0.073)$ (Figure 1), but no correlation with age, body mass index, blood pressure (systolic, diastolic), LDL-C, TG, FPG, IRI or homeostasis model assessment insulin resistance index (Table 2) was found. There were no significant correlations of morning, midnight or post-1 mg dexamethasone suppression test cortisol levels with any of the clinical parameters (data not shown).

To examine whether surgical or medical treatment affects cardiovascular risk factors, changes in the number of risk factors during the follow-up period were compared between the OP and non-OP groups. The number of cardiovascular risk factors tended to increase, although this trend was insignificant $(P=0.096$, from $2[0.25-2]$ to $2[1.25-3])$ in the non-OP group. The number of cardiovascular risk factors decreased significantly $(P=0.020$, from $2[1.25-3]$ to $1[0-2])$ in the OP group (Figure 2).

To further determine which cardiovascular risk factor(s) are responsible for the decreased number of risk factors, changes in clinical outcome (hypertension, dyslipidemia, IGT/DM and obesity) during the follow-up period were compared between the non-OP and OP groups. Significant improvements in hypertension $(P=0.005)$, dyslipidemia $(P=0.044)$ and IGT/DM $(P=0.025)$, but not obesity $(P=0.085)$, were observed in the OP group compared with the nonOP group (Figure 3).

\section{DISCUSSION}

The present study clearly showed a high prevalence of cardiovascular risk factors and significant correlations of 24-h UFC excretions with HbAlc and HDL-C levels in our SCS patients. The present study further revealed the number of cardiovascular risk factors in SCS patients significantly decreased with improvements in hypertension, dyslipidemia and IGT/DM in the OP group but not in the non-OP group. 
Table 1 Characteristics of 16 patients with subclinical Cushing's syndrome

\begin{tabular}{|c|c|c|c|}
\hline & Total (16) & OP group (8) & Non-OP group (8) \\
\hline Age (years) & $56.6 \pm 11.3$ & $55.9 \pm 10.5$ & $57.2 \pm 12.5$ \\
\hline \multicolumn{4}{|l|}{ Risk factors } \\
\hline Hypertension (\%) & $9(56)$ & $5(63)$ & $4(50)$ \\
\hline Diabetes/IGT (\%) & $4(25) / 4(25)$ & $3(38) / 3(38)$ & $1(13) / 1(13)$ \\
\hline Body mass index $\left(\mathrm{kg} \mathrm{m}^{-2}\right)$ & $23.7 \pm 4.1$ & $25.0 \pm 4.5$ & $22.6 \pm 3.6$ \\
\hline Systolic blood pressure (mm Hg) & $127 \pm 18$ & $133 \pm 21$ & $122 \pm 13$ \\
\hline Diastolic blood pressure $(\mathrm{mm} \mathrm{Hg})$ & $81 \pm 11$ & $84 \pm 12$ & $78 \pm 9$ \\
\hline HDL-cholesterol $\left(\mathrm{mg} \mathrm{dl}^{-1}\right)$ & $68 \pm 32$ & $64 \pm 22$ & $73 \pm 41$ \\
\hline LDL-cholesterol (mg dl-1) & $101 \pm 29$ & $102 \pm 30$ & $99 \pm 30$ \\
\hline HOMA-R & $1.29(0.92-2.00)$ & $1.53(1.03-2.43)$ & $0.93(0.90-1.93)$ \\
\hline \multicolumn{4}{|l|}{ Serum cortisol $\left(\mu \mathrm{g} \mathrm{d} \mathrm{l}^{-1}\right)$} \\
\hline \multicolumn{4}{|l|}{ Circadian rhythm } \\
\hline 0800 & $15.0 \pm 4.7$ & $13.9 \pm 2.6$ & $16.0 \pm 4.0$ \\
\hline 2300 & $8.7 \pm 4.7$ & $8.8 \pm 4.0$ & $8.7 \pm 5.6$ \\
\hline DST 0800 & $7.5 \pm 5.1$ & $8.0 \pm 5.1$ & $7.0 \pm 5.4$ \\
\hline Plasma ACTH (pg ml-1; reference range: $10-60$ ) & $9.1 \pm 4.9$ & $9.3 \pm 5.5$ & $8.8 \pm 4.6$ \\
\hline 24-h UFC excretion ( $\mu$ g per day; reference range: $30-80$ ) & $42.0(25.0-67.4)$ & $46.0(29.5-95.0)$ & $34.1(15.3-52.0)$ \\
\hline DHEA-S (ng dl-1) & $268 \pm 222$ & $169 \pm 120^{*}$ & $559 \pm 164$ \\
\hline Tumor size (mm) & $26.3 \pm 8.7$ & $25.5 \pm 6.8$ & $27.1 \pm 10.1$ \\
\hline
\end{tabular}

Abbreviations: DHEA-S, dehydroepiandrosterone sulfate; DST, dexamethasone suppression test; HDL, high-density lipoprotein; HOMA-R, homeostasis model assessment insulin resistance index; IGT, impaired glucose tolerance; LDL, low-density lipoprotein; non-OP, non-operated; OP, operated; UFC, urinary free-cortisol excretion.

Data mean \pm s.d., or median ( $25-75$ percentile)

${ }^{*} P<0.05$ vs. non-operated group.
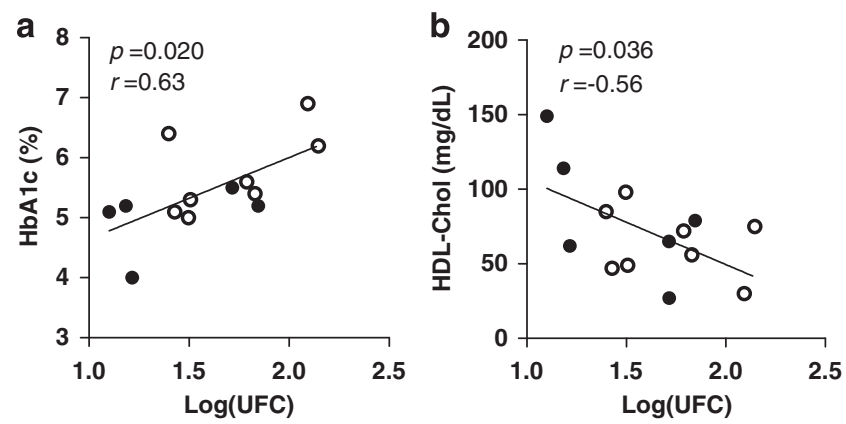

Figure 1 Correlations of 24-h urinary free-cortisol (UFC) excretion with $\mathrm{HbAlc}$ and high-density lipoprotein-cholesterol (HDL-C) in 16 subclinical Cushing's syndrome patients. Correlations between 24-h UFC excretion and (a) HbAlc levels and (b) HDL-C levels in the non-operated group (•) and the operated group $(O)$.

The prevalence of IGT/DM (50\%) in our SCS patients (male: $29 \%$, female: $21 \%$ ) was higher than in the general Japanese population (50-59 y.o), whereas the prevalence of hypertension (56\%) and obesity $(19 \%)$ in our series was comparable with that in the general population in Japan: hypertension, $51 \%$ among males and $41 \%$ among females; and obesity, 31\% among males and 21\% among females. ${ }^{19}$ Notably, $75 \%$ of our SCS patients exhibited two or more cardiovascular risk factors, suggesting the accumulation of cardiovas-
Table 2 Correlations of 24-h UFC excretion and body mass index, blood pressure and various metabolic parameters in 16 SCS patients

\begin{tabular}{lcl}
\hline & Peason's (r) & P-value \\
\hline Age & -0.24 & 0.41 \\
Body mass index & 0.028 & 0.92 \\
Systolic blood pressure & 0.16 & 0.59 \\
Diastolic blood pressure & 0.35 & 0.22 \\
HDL-cholesterol & -0.56 & 0.036 \\
LDL-cholesterol & 0.024 & 0.93 \\
Triglyceride & 0.29 & 0.32 \\
Fasting plasma glucose & 0.49 & 0.073 \\
Fasting insulin & -0.39 & 0.27 \\
Log (HOMA-R) & 0.29 & 0.39 \\
HbA1c & 0.63 & 0.020 \\
\hline
\end{tabular}

Abbreviations: HDL, high-density lipoprotein; HOMA-R, homeostasis model assessment insulin resistance index; HbAlc: hemoglobin A1c; LDL, low-density lipoprotein; SCS, subclinical Cushing's syndrome; UFC, urinaryfree-cortisol excretion.

$r$, the coefficient of Person's correlation.

cular risk factors in SCS. Our data are in agreement with those of previous studies, ${ }^{6-8,11,20,21}$ as well as a recent report from Japan. ${ }^{12}$ Taken together, these data suggest that even a mild cortisol excess in SCS could have a crucial role in the accumulation of cardiovascular risk factors. 


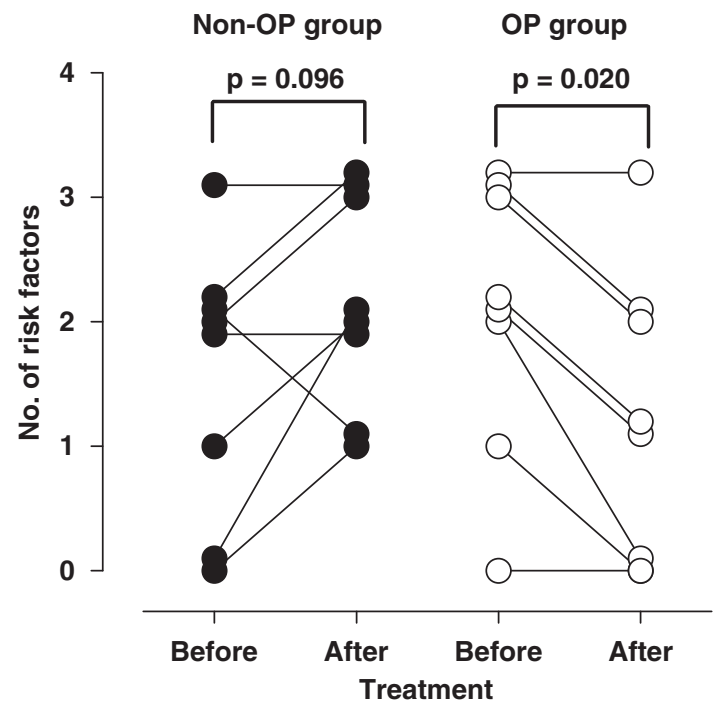

Figure 2 Changes in the number of cardiovascular risk factors in nonoperated (non-OP) and operated (OP) subclinical Cushing's syndrome patients over the follow-up period. Number of cardiovascular risk factors in the non-OP group $(\bullet: n=8)$ and OP group $(O: n=8)$ before and after follow-up.

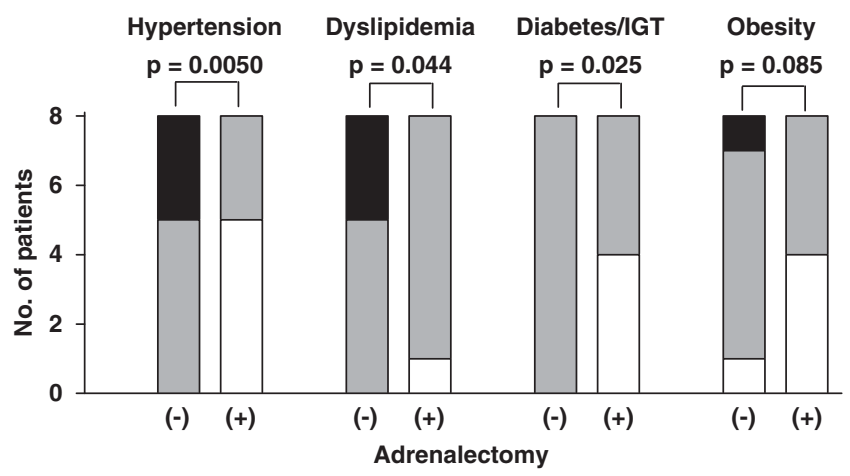

Figure 3 Comparison of clinical outcome of cardiovascular risk factors between non-operated and operated subclinical Cushing's syndrome patients during the follow-up period. Each column shows clinical outcomes ( $\square$ : improvement, $\square$ : no change, $\mathbf{\square}$ : deterioration) of cardiovascular risk factors (hypertension, dyslipidemia, diabetes/impaired glucose tolerance (IGT) and obesity) in the non-operated $(n=8)$ and operated $(n=8)$ groups. A statistical comparison between the two groups is shown.

The present study clearly demonstrated that 24-h UFC excretion, a reliable index of daily cortisol production, positively correlated with HbA1c levels and negatively with HDL-C levels in our SCS patients. Because chronic glucocorticoid excess is associated with insulin resistance ${ }^{22}$ and decreased HDL-C levels ${ }^{23,24}$ in overt CS patients, it is suggested that even the mild cortisol excess in SCS could contribute to impaired glucose and lipid metabolism. In agreement with a previous study, ${ }^{12}$ midnight cortisol and post-dexamethasone suppression test cortisol levels (both indices of autonomous cortisol secretion) did not correlate with any of the clinical parameters in our SCS patients. We have recently shown that excessive rather than autonomous cortisol secretion is more closely associated with endothelial dysfunction, an early stage of atherosclerosis, in overt CS patients. This condition is reversible after correction of hypercortisolemia. ${ }^{25}$ Thus, a mild excess of cortisol secretion, as reflected by 24 -h UFC excretion, is likely to affect glycemic and lipid metabolism in SCS patients. It is therefore suggested that SCS patients with higher UFC excretion represent potential candidates for surgical treatment. However, because of the limited number of patients included in our series, the extent to which UFC excretion is causally related to abnormal glycemic and lipid metabolism in SCS patients remains to be determined.

In the present study, the number of cardiovascular risk factors significantly decreased in SCS patients treated with adrenalectomy but not in patients subjected to conservative treatment. Our data complement those reported by other studies. ${ }^{12,13}$ Thus, surgical treatment could be beneficial among SCS patients, serving to improve cardiovascular morbidity and/or mortality by decreasing the cardiovascular risk factors possibly resulting from correction of a mild cortisol excess.

It remains controversial whether cardiovascular risk factors in SCS patients could improve after adrenalectomy. Several studies have reported that hypertension improved after adrenalectomy in SCS patients, ${ }^{8,11-13}$ whereas some studies have shown that adrenalectomy did not significantly improve the CVD risk profile. ${ }^{26,27}$ The present study, however, showed that not only hypertension but also DM/IGT and dyslipidemia significantly improved in our SCS patients after the removal of adrenal tumors. Our data are in accordance with those of a recent study showing that blood pressure, weight and fasting glucose in SCS patients improved after adrenalectomy. ${ }^{28}$ These findings lend strong support to the contention that surgical removal of adrenal tumors could be a therapeutic option for SCS patients who present with multiple cardiovascular risk factors, such as hypertension, IGT/ $\mathrm{DM}$ and dyslipidemia. However, larger prospective clinical studies are required to address the key issue, that is, whether surgical treatment can reduce cardiovascular morbidity and mortality in SCS patients over the long term.

The present study carries certain limitations. First, because of the retrospective nature of the study, we could not match the baseline characteristics of the non-OP group and the OP group with higher $\mathrm{HbAlc}$ and lower dehydroepiandrosterone sulfate levels. Second, because we could not standardize the treatment regimes for hypertension, diabetes and dyslipidemia, it is difficult to differentiate the influence of surgical intervention from the effects of various treatments involving lifestyle modifications, such as diet and exercise, medications and patient compliance. Third, because of the limited number of SCS patients, we could not perform multivariate analysis to identify any independent determinant(s) of indication and outcome of treatment. A prospective study with a larger sample size is required.

In conclusion, the present study demonstrated the increased prevalence of cardiovascular risk factors among SCS patients as well as a positive correlation between mild hypercortisolism and impaired glucose/lipid metabolism. Adrenalectomy significantly improved hypertension, dyslipidemia and diabetes/IGT in SCS patients, suggesting the possible involvement of mild hypercortisolism in the development of multiple cardiovascular risk factors in SCS.

\section{CONFLICT OF INTEREST}

The authors declare no conflict of interest.

\section{ACKNOWLEDGEMENTS}

This study was supported in part by Grants-in-Aid from the Ministry of Education, Science, Sports, Culture and Technology and the Ministry of Health, Labor and Welfare of Japan. 
1 Reincke M. Subclinical Cushing's syndrome. Endocrinol Metab Clin North Am 2000; 29: 43-56.

2 Terzolo M, Osella G, Ali A, Borretta G, Cesario F, Paccotti P, Angeli A. Subclinical Cushing's syndrome in adrenal incidentaloma. Clin Endocrinol (Oxf) 1998; 48: 89-97.

3 Terzolo M, Bovio S, Pia A, Reimondo G, Angeli A. Management of adrenal incidentaloma. Best Pract Res Clin Endocrinol Metab 2009; 23: 233-243.

4 Bovio S, Cataldi A, Reimondo G, Sperone P, Novello S, Berruti A, Borasio P, Fava C, Dogliotti L, Scagliotti GV, Angeli A, Terzolo M. Prevalence of adrenal incidentaloma in a contemporary computerized tomography series. J Endocrinol Invest 2006; 29: 298-302.

5 Mantero F, Terzolo M, Arnaldi G, Osella G, Masini AM, Ali A, Giovagnetti M, Opocher G, Angeli A. A survey on adrenal incidentaloma in Italy. Study group on adrenal tumors of the italian society of endocrinology. J Clin Endocrinol Metab 2000; 85: 637-644.

6 Reincke M, Nieke J, Krestin GP, Saeger W, Allolio B, Winkelmann W. Preclinical Cushing's syndrome in adrenal 'incidentalomas': comparison with adrenal Cushing's syndrome. J Clin Endocrinol Metab 1992; 75: 826-832.

7 Rossi R, Tauchmanova L, Luciano A, Di Martino M, Battista C, Del Viscovo L, Nuzzo V, Lombardi G. Subclinical Cushing's syndrome in patients with adrenal incidentaloma: clinical and biochemical features. J Clin Endocrinol Metab 2000; 85: 1440-1448.

8 Tauchmanova L, Rossi R, Biondi B, Pulcrano M, Nuzzo V, Palmieri EA, Fazio S, Lombardi G. Patients with subclinical Cushing's syndrome due to adrenal adenoma have increased cardiovascular risk. J Clin Endocrinol Metab 2002; 87: 4872-4878.

9 Etxabe J, Vazquez JA. Morbidity and mortality in Cushing's disease: an epidemiological approach. Clin Endocrinol (Oxf) 1994; 40: 479-484.

10 Arnaldi G, Angeli A, Atkinson AB, Bertagna X, Cavagnini F, Chrousos GP, Fava GA Findling JW, Gaillard RC, Grossman AB, Kola B, Lacroix A, Mancini T, Mantero F, Newell-Price J, Nieman LK, Sonino N, Vance ML, Giustina A, Boscaro M. Diagnosis and complications of Cushing's syndrome: a consensus statement. J Clin Endocrinol Metab 2003; 88: 5593-5602.

11 Erbil Y, Ademoglu E, Ozbey N, Barbaros U, Yanik BT, Salmaslioglu A, Bozbora A, Ozarmagan S. Evaluation of the cardiovascular risk in patients with subclinical Cushing syndrome before and after surgery. World J Surg 2006; 30: 1665-1671.

12 Tsuiki M, Tanabe A, Takagi S, Naruse M, Takano K. Cardiovascular risks and their longterm clinical outcome in patients with subclinical Cushing's syndrome. Endocr J 2008; 55: 737-745.

13 Toniato A, Merante-Boschin I, Opocher G, Pelizzo MR, Schiavi F, Ballotta E. Surgical versus conservative management for subclinical Cushing syndrome in adrenal incidentalomas: a prospective randomized study. Ann Surg 2009; 249: 388-391.

14 Stewart PM. Is subclinical Cushing's syndrome an entity or a statistical fallout from diagnostic testing? Consensus surrounding the diagnosis is required before optimal treatment can be defined. J Clin Endocrinol Metab 2010; 95: 2618-2620.

15 Nawata H, Demura H, Suda T, Takayanagi R. Adrenal preclinical Cushing's syndrome, In Annual Report of the Ministry of Health and Welfare 'Disorders of Adrenal Hormones' Research Committee, Japan, 1996, 223-226.
16 Ogihara T, Saruta T, Rakugi H, Fujimoto A, Ueshima K, Yasuno S, Oba K, Takeda K, Higaki J, Nakao K. Relationship between the achieved blood pressure and the incidence of cardiovascular events in Japanese hypertensive patients with complications: a sub-analysis of the CASE-J trial. Hypertens Res 2009; 32: 248-254.

17 Seino Y, Nanjo K, Tajima N, Kadowaki T, Kashiwagi A, Araki E, Ito C, Inagaki N, Iwamoto Y, Kasuga M, Hanafusa T, Haneda M, Ueki K. Report of the committee on the classification and diagnostic criteria of diabetes mellitus. J Jpn Diabe Soc 2010; 53: 450-467.

18 Teramoto T, Sasaki J, Ueshima H, Egusa G, Kinoshita M, Shimamoto K, Daida H, Biro S, Hirobe K, Funahashi T, Yokote K, Yokode M. Diagnostic criteria for dyslipidemia. Executive summary of Japan Atherosclerosis Society (JAS) guideline for diagnosis and prevention of atherosclerotic cardiovascular diseases for Japanese. $J$ Atheroscler Thromb 2007; 14: 155-158.

19 Health and Welfare Statistics Association. Journal of Health and Welfare Statistics 2010/2011 2010; 57: 79-82.

20 Bernini G, Moretti A, lacconi P, Miccoli P, Nami R, Lucani B, Salvetti A. Anthropometric, haemodynamic, humoral and hormonal evaluation in patients with incidental adrenocortical adenomas before and after surgery. Eur J Endocrinol 2003; 148: 213-219.

21 Mitchell IC, Auchus RJ, Juneja K, Chang AY, Holt SA, Snyder III WH, Nwariaku FE. 'Subclinical Cushing's syndrome' is not subclinical improvement after adrenalectomy in 9 patients. Surgery 2007; 142: 900-905.

22 Lambillotte C, Gilon P, Henquin JC. Direct glucocorticoid inhibition of insulin secretion. An in vitro study of dexamethasone effects in mouse islets. J Clin Invest 1997; 99: 414-423.

23 Colao A, Pivonello R, Spiezia S, Faggiano A, Ferone D, Filippella M, Marzullo P, Cerbone G, Siciliani M, Lombardi G. Persistence of increased cardiovascular risk in patients with Cushing's disease after five years of successful cure. J Clin Endocrinol Metab 1999; 84: 2664-2672.

24 Weigensberg MJ, Toledo-Corral CM, Goran MI. Association between the metabolic syndrome and serum cortisol in overweight Latino youth. J Clin Endocrinol Metab 2008; 93: 1372-1378.

25 Akaza I, Yoshimoto T, Tsuchiya K, Hirata Y. Endothelial dysfunction associated with hypercortisolism is reversible in Cushing's syndrome. Endocr J 2010; 57: 245-252.

26 Sereg M, Szappanos A, Toke J, Karlinger K, Feldman K, Kaszper E, Varga I, Glaz E, Racz K, Toth M. Atherosclerotic risk factors and complications in patients with nonfunctioning adrenal adenomas treated with or without adrenalectomy: a long-term follow-up study. Eur J Endocrinol 2009; 160: 647-655.

27 Giordano R, Marinazzo E, Berardelli R, Picu A, Maccario M, Ghigo E, Arvat E. Longterm morphological, hormonal, and clinical follow-up in a single unit on 118 patients with adrenal incidentalomas. Eur J Endocrinol 2010; 162: 779-785.

28 Chiodini I, Morelli V, Salcuni AS, Eller-Vainicher C, Torlontano M, Coletti F, lorio L, Cuttitta A, Ambrosio A, Vicentini L, Pellegrini F, Copetti M, Beck-Peccoz P, Arosio M, Ambrosi B, Trischitta V, Scillitani A. Beneficial metabolic effects of prompt surgical treatment in patients with an adrenal incidentaloma causing biochemical hypercortisolism. J Clin Endocrinol Metab 2010; 95: 2736-2745. 